\title{
Mass composition of Telescope Array's surface detectors events using deep learning
}

\author{
I. Kharuk ${ }^{a, b, *}$ and O. Kalashev ${ }^{a, b}$ on behalf of the Telescope Array Collaboration \\ (a complete list of authors can be found at the end of the proceedings) \\ ${ }^{a}$ Institute for Nuclear Research of the Russian Academy of Sciences, \\ 60th October Anniversary Prospect, 7a, Moscow, 117312, Russia \\ ${ }^{b}$ Moscow Institute of Physics and Technology, \\ Institutsky lane 9, Dolgoprudny, Moscow region, 141700, Russia \\ E-mail: ivan.kharuk@phystech.edu
}

We report on an improvement of deep learning techniques used for identifying primary particles of atmospheric air showers. The progress was achieved by using two neural networks. The first works as a classifier for individual events, while the second predicts fractions of elements in an ensemble of events based on the inference of the first network. For a fixed hadronic model, this approach yields an accuracy of 90\% in identifying fractions of elements in an ensemble of events.

$37^{\text {th }}$ International Cosmic Ray Conference (ICRC 2021)

July 12th - 23rd, 2021

Online - Berlin, Germany

\footnotetext{
${ }^{*}$ Presenter
} 


\section{Introduction}

Machine learning techniques are widely used for identifying primary particle air showers caused by cosmic rays [1-3]. The typical problem one faces in this area is the high variance of the data measured by detectors. Namely, since the evolution of an air shower is a highly stochastic process, detectors readings are subject to large fluctuations. The situation is complicated further by the presence of noise in the data, sparse detectors grid, and defective and offline detectors. These are the reasons why all of the developed techniques have rather high error rate in estimating the mass of the primary particle.

We suggest an improvement to the existing techniques by switching from individual predictions to estimating the fractions of elements in an ensemble of events. The corresponding procedure includes two steps. Given an ensemble of events, we first use a neural network, which we dub the classifier, to predict the primary particle for each of the individual events. At the second step, we average the obtained predictions and pass them through another neural network, the converter, which yields true portions of elements in the ensemble. The resulting scheme is presented on figure 1 , and allows us to reach an error rate of less than $10 \%$ in obtaining the fractions of elements in an ensemble. Below we describe the steps of our data analysis in detail.

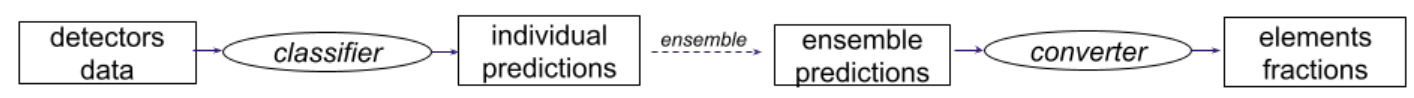

Figure 1: Scheme of analyzing the data.

\section{Classifier}

The classifier is trained to predict the primary particle for an individual event. The training data is the Monte-Carlo simulated data for the Telescope Array surface detectors readings [4], passing quality cuts on the events (see [1] for their description). The data includes protons, helium, nitrogen, and iron as primary particles, and air shower evolution was modeled with QGSJET II-03 hadronic model [5]. For illustration purposes, here we consider the events only in the energy window between $10^{18.75} \mathrm{eV}$ and $10^{19} \mathrm{eV}$ (roughly 100000 events in total). The architecture of the classifier consists of 3 blocks, each of which is specialized for analyzing certain kind of data.

The first block is the spatial detector bundle. For each of the events we introduce a six by six square grid of detectors centered on the shower core. Each element of the grid stores the following data: coordinates of the detector, total signal, time of the flat front arrival, relative starting time of detectors readings, and flags related to detector status. This data is analyzed by series of convolution layers, yielding spatial features of an event as the output.

The second block analyzes the waveform from the detector with the largest integral signal. By passing the waveform though convolution layers, we obtain the features of the largest signal.

The third block is a temporal detector bundle. For a given event, we collect all triggered detectors and order them according to the time of the plane front arrival. We first pass each of the recorded waveforms though series of recurrent cells to extract their features. These features are 
further analyzed by another set of recurrent cells, according to their order in the temporal chain. Thus we obtain temporal features of the event.

As the last step, we concatenate all of the obtained features and supplement them with composition-sensitive reconstruction parameters. Together, they are passed through a set of fully connected dense layers. The output of these layers, and thus of the whole neural network, are the probabilities of the primary particle being proton, helium, nitrogen, and iron. ${ }^{1}$

\section{Converter}

For the second stage, we take an ensemble of events and obtain predictions for each of them by passing the corresponding data through the classifier. The average of these predictions can be considered as the estimation of the fractions of elements in the ensemble. For an arbitrary ensemble, the typical predictions are depicted on the second section of figure 2 .

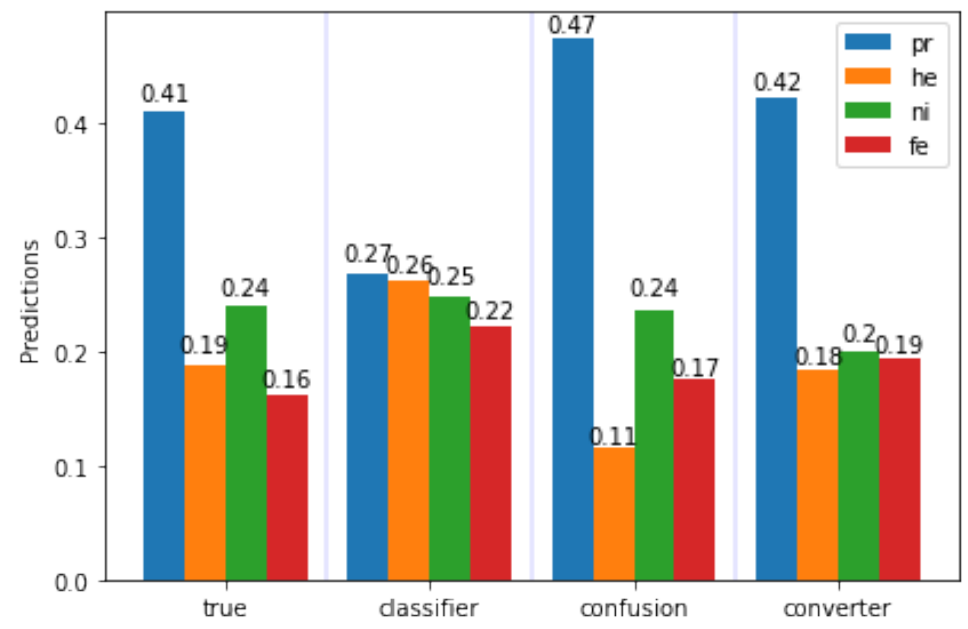

Figure 2: Predictions of the fractions of elements in an ensemble of events using various approaches. From left to right: true fractions of elements in the ensemble; averaged predictions of the classifier; predictions improved by using the confusion matrix; converter predictions.

As one can see, the averaged predictions of the classifier are rather far from the true values. To improve them, one can make use of the confusion matrix, which provides information on how wrong the predictions are in average. This allows for correction of the predictions by multiplying them by the inverse of the confusion matrix. As it can be seen from the third section of figure 2, this approach yields much better predictions.

One can, however, improve the predictions even further. The limitations of the confusion matrix approach is that it provides linear transformation of the predictions, which works best for ensembles consisting of one sort of primary particles only. These limitations can be avoided by introducing another neural network, the converter, which is trained to map averaged predictions of the classifier to their true values. The predictions obtained in this way are depicted on the last section of figure 2 , and provide the greatest accuracy yet.

\footnotetext{
${ }^{1}$ Any of the blocks can be used without others for predicting the primary particle type. However, dropping any of them results in a much lower accuracy of the predictions.
} 


\begin{tabular}{|c|c|c|c|c|}
\hline & proton & helium & nitrogen & iron \\
\hline classifier & 0.10 & 0.11 & 0.11 & 0.09 \\
\hline confusion & 0.06 & 0.14 & 0.12 & 0.04 \\
\hline converter & 0.03 & 0.07 & 0.06 & 0.02 \\
\hline
\end{tabular}

Table 1: Mean absolute errors of the methods for different particles.

To compare different approaches, we evaluated their mean absolute error on 2000 ensembles, each consisting of 5000 individual events. The results are presented in table 1 . Of the different methods, the analysis using two neural networks yields best result, with an error rate of just $7 \%$.

\section{Model dependence}

To estimate the error associated with the choice of the hadronic model, we analyzed the predictions made for Monte-Carlo simulation with QGSJET II-04 model. Namely, we made "pure" ensembles consisting of proton, helium, nitrogen, and iron primaries and analyzed them using the scheme described above. The resulting classifier predictions are shown on figure 3. The masses of primary particles are strongly overestimated. For example, nitrogen is recognized as pure iron by the classifier-converter chain. Because of the large model error we did not apply this method to the actual data.

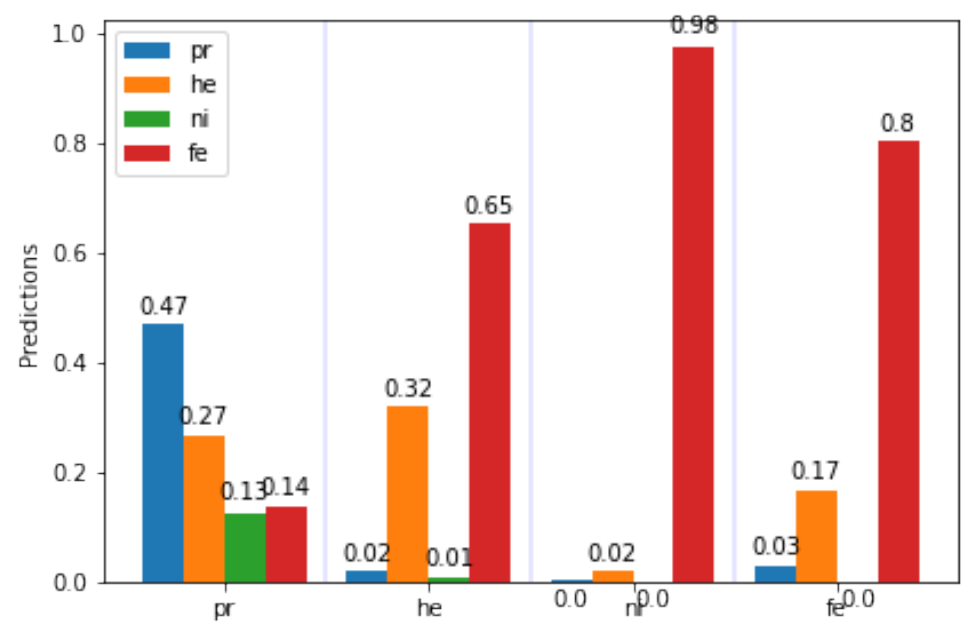

Figure 3: Predictions for "pure" ensembles modeled with QGSJET II-04 hadronic model. From left to right: the converter predictions for protons, helium, nitrogen, and iron, respectively.

\section{Conclusion}

The introduced method of analyzing data by the chain of two neural networks allows us to achieve very high accuracy in estimating the fractions of elements in an ensemble of events. However, while gaining such high accuracy, the method became very sensitive to the hadronic 
model used in the Monte-Carlo simulations. As a consequence, our method has a large model error. We hope to overcome this problem in future by training neural networks on multiple ensembles of events, simulated with various hadronic models.

\section{Acknowledgements:}

The Telescope Array experiment is supported by the Japan Society for the Promotion of Science(JSPS) through Grants-in-Aid for Priority Area 431, for Specially Promoted Research JP21000002, for Scientific Research (S) JP19104006, for Specially Promoted Research JP15H05693, for Scientific Research (S) JP15H05741 and JP19H05607, for Science Research (A) JP18H03705, for Young Scientists (A) JPH26707011, and for Fostering Joint International Research (B) JP19KK0074, by the joint research program of the Institute for Cosmic Ray Research (ICRR), The University of Tokyo; by the Pioneering Program of RIKEN for the Evolution of Matter in the Universe (r-EMU); by the U.S. National Science Foundation awards PHY-1404495, PHY-1404502, PHY-1607727, PHY-1712517, PHY-1806797 and PHY-2012934; by the National Research Foundation of Korea (2017K1A4A3015188, 2020R1A2C1008230, \& 2020R1A2C2102800) ; the development and application of the machine learning analysis method is supported by the Russian Science Foundation grant No. 17-72-20291 (INR), IISN project No. 4.4501.18, and Belgian Science Policy under IUAP VII/37 (ULB). This work was partially supported by the grants ofThe joint research program of the Institute for Space-Earth Environmental Research, Nagoya University and Inter-University Research Program of the Institute for Cosmic Ray Research of University of Tokyo. The foundations of Dr. Ezekiel R. and Edna Wattis Dumke, Willard L. Eccles, and George S. and Dolores Doré Eccles all helped with generous donations. The State of Utah supported the project through its Economic Development Board, and the University of Utah through the Office of the Vice President for Research. The experimental site became available through the cooperation of the Utah School and Institutional Trust Lands Administration (SITLA), U.S. Bureau of Land Management (BLM), and the U.S. Air Force. We appreciate the assistance of the State of Utah and Fillmore offices of the BLM in crafting the Plan of Development for the site. Patrick A. Shea assisted the collaboration with valuable advice and supported the collaboration's efforts. The people and the officials of Millard County, Utah have been a source of steadfast and warm support for our work which we greatly appreciate. We are indebted to the Millard County Road Department for their efforts to maintain and clear the roads which get us to our sites. We gratefully acknowledge the contribution from the technical staffs of our home institutions. An allocation of computer time from the Center for High Performance Computing at the University of Utah is gratefully acknowledged.

\section{References}

[1] R. Abbasi, M. Abe, T. Abu-Zayyad, M. Allen, R. Azuma, E. Barcikowski et al., Mass composition of ultrahigh-energy cosmic rays with the telescope array surface detector data, Physical Review D 99 (2019) 022002.

[2] D. Ivanov, O. E. Kalashev, M. Y. Kuznetsov, G. I. Rubtsov, T. Sako, Y. Tsunesada et al., Using Deep Learning to Enhance Event Geometry Reconstruction for the Telescope Array Surface Detector, 2005 . 07117.

[3] Pierre Auger collaboration, A. Aab et al., Deep-learning based reconstruction of the shower maximum $x_{\max }$ using the water-cherenkov detectors of the pierre auger observatory, 2101.02946.

[4] H. Tokuno, T. Abu-Zayyad, R. Aida, M. Allen, R. Azuma, E. Barcikowski et al., The status of the telescope array experiment, in Journal of Physics: Conference Series, vol. 293, p. 012035, IOP Publishing, 2011.

[5] S. Ostapchenko, Qgsjet-ii: towards reliable description of very high energy hadronic interactions, Nuclear Physics B-Proceedings Supplements 151 (2006) 143-146. 


\section{Full Authors List: Telescope Array Collaboration}

R.U. Abbasi ${ }^{1,2}$, T. Abu-Zayyad ${ }^{1,2}$, M. Allen ${ }^{2}$, Y. Arai $^{3}$, R. Arimura ${ }^{3}$, E. Barcikowski ${ }^{2}$, J.W. Belz ${ }^{2}$, D.R. Bergman ${ }^{2}$, S.A. Blake ${ }^{2}$, I. Buckland ${ }^{2}$, R. $\mathrm{Cady}^{2}$, B.G. Cheon ${ }^{4}$, J. Chiba ${ }^{5}$, M. Chikawa ${ }^{6}$, T. Fujii ${ }^{7}$, K. Fujisue ${ }^{6}$, K. Fujita ${ }^{3}$, R. Fujiwara ${ }^{3}$, M. Fukushima ${ }^{6}$, R. Fukushima ${ }^{3}$, G. Furlich ${ }^{2}$, R. Gonzalez ${ }^{2}$, W. Hanlon ${ }^{2}$, M. Hayashi ${ }^{8}$, N. Hayashida ${ }^{9}$, K. Hibino ${ }^{9}$, R. Higuchi ${ }^{6}$, K. Honda $^{10}$, D. Ikeda ${ }^{9}$, T. Inadomi ${ }^{11}$, N. Inoue ${ }^{12}$, T. Ishii ${ }^{10}$, H. Ito ${ }^{13}$, D. Ivanov ${ }^{2}$, H. Iwakura ${ }^{11}$, A. Iwasaki ${ }^{3}$, H.M. Jeong ${ }^{14}$, S. Jeong ${ }^{14}$, C.C.H. Jui ${ }^{2}$, K. Kadota ${ }^{15}$, F. Kakimoto ${ }^{9}$, O. Kalashev ${ }^{16}$, K. Kasahara ${ }^{17}$, S. Kasami ${ }^{18}$, H. Kawai ${ }^{19}$, S. Kawakami ${ }^{3}$, S. Kawana ${ }^{12}$, K. Kawata ${ }^{6}$, I. Kharuk ${ }^{16}$, E. Kido ${ }^{13}$, H.B. Kim ${ }^{4}$, J.H. Kim ${ }^{2}$, J.H. Kim ${ }^{2}$, M.H. Kim ${ }^{14}$, S.W. Kim ${ }^{14}$, Y. Kimura ${ }^{3}$, S. Kishigami ${ }^{3}$, Y. Kubota ${ }^{11}$, S. Kurisu ${ }^{11}$, V. Kuzmin ${ }^{16 *}$, M. Kuznetsov ${ }^{16,20}$, Y.J. Kwon ${ }^{21}$, K.H. Lee ${ }^{14}$, B. Lubsandorzhiev ${ }^{16}$, J.P. Lundquist ${ }^{2,22}$, K. Machida ${ }^{10}$, H. Matsumiya ${ }^{3}$, T. Matsuyama ${ }^{3}$, J.N. Matthews ${ }^{2}$, R. Mayta ${ }^{3}$, M. Minamino ${ }^{3}$, K. Mukai ${ }^{10}$, I. Myers ${ }^{2}$, S. Nagataki ${ }^{13}$, K. Nakai ${ }^{3}$, R. Nakamura ${ }^{11}$, T. Nakamura ${ }^{23}$, T. Nakamura ${ }^{11}$, Y. Nakamura ${ }^{11}$, A. Nakazawa ${ }^{11}$, E. Nishio ${ }^{18}$, T. Nonaka ${ }^{6}$, H. Oda ${ }^{3}$, S. Ogio ${ }^{3,24}$, M. Ohnishi ${ }^{6}$, H. Ohoka ${ }^{6}$, Y. Oku ${ }^{18}$, T. Okuda ${ }^{25}$, Y. Omura ${ }^{3}$, M. Ono ${ }^{13}$, R. Onogi ${ }^{3}$, A. Oshima ${ }^{3}$, S. Ozawa ${ }^{26}$, I.H. Park ${ }^{14}$, M. Potts ${ }^{2}$, M.S. Pshirkov ${ }^{16,27}$, J. Remington ${ }^{2}$, D.C. Rodriguez ${ }^{2}$, G.I. Rubtsov ${ }^{16}$, D. Ryu ${ }^{28}$, H. Sagawa ${ }^{6}$, R. Sahara ${ }^{3}$, Y. Saito ${ }^{11}$, N. Sakaki ${ }^{6}$, T. Sako ${ }^{6}$, N. Sakurai ${ }^{3}$, K. Sano ${ }^{11}$, K. Sato ${ }^{3}$, T. Seki ${ }^{11}$, K. Sekino ${ }^{6}$, P.D. Shah ${ }^{2}$, Y. Shibasaki ${ }^{11}$, F. Shibata ${ }^{10}$, N. Shibata ${ }^{18}$, T. Shibata ${ }^{6}$, H. Shimodaira ${ }^{6}$, B.K. Shin ${ }^{28}$, H.S. Shin ${ }^{6}$, D. Shinto ${ }^{18}$, J.D. Smith ${ }^{2}$, P. Sokolsky ${ }^{2}$, N. Sone ${ }^{11}$, B.T. Stokes ${ }^{2}$, T.A. Stroman ${ }^{2}$, Y. Takagi $^{3}$,

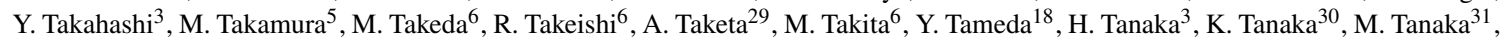
Y. Tanoue ${ }^{3}$, S.B. Thomas ${ }^{2}$, G.B. Thomson ${ }^{2}$, P. Tinyakov ${ }^{16,20}$, I. Tkachev ${ }^{16}$, H. Tokuno ${ }^{32}$, T. Tomida ${ }^{11}$, S. Troitsky ${ }^{16}$, R. Tsuda ${ }^{3}$, Y. Tsunesada ${ }^{3,24}$, Y. Uchihori ${ }^{33}$, S. Udo ${ }^{9}$, T. Uehama ${ }^{11}$, F. Urban ${ }^{34}$, T. Wong ${ }^{2}$, K. Yada $^{6}$, M. Yamamoto ${ }^{11}$, K. Yamazaki ${ }^{9}$, J. Yang ${ }^{35}$, K. Yashiro ${ }^{5}$, F. Yoshida ${ }^{18}$, Y. Yoshioka ${ }^{11}$, Y. Zhezher ${ }^{6,16}$, and Z. Zundel ${ }^{2}$

${ }^{1}$ Department of Physics, Loyola University Chicago, Chicago, Illinois, USA

${ }^{2}$ High Energy Astrophysics Institute and Department of Physics and Astronomy, University of Utah, Salt Lake City, Utah, USA

${ }^{3}$ Graduate School of Science, Osaka City University, Osaka, Osaka, Japan

${ }^{4}$ Department of Physics and The Research Institute of Natural Science, Hanyang University, Seongdong-gu, Seoul, Korea

${ }^{5}$ Department of Physics, Tokyo University of Science, Noda, Chiba, Japan

${ }^{6}$ Institute for Cosmic Ray Research, University of Tokyo, Kashiwa, Chiba, Japan

${ }^{7}$ The Hakubi Center for Advanced Research and Graduate School of Science, Kyoto University, Kitashirakawa-Oiwakecho, Sakyo-ku, Kyoto, Japan

${ }^{8}$ Information Engineering Graduate School of Science and Technology, Shinshu University, Nagano, Nagano, Japan

${ }^{9}$ Faculty of Engineering, Kanagawa University, Yokohama, Kanagawa, Japan

${ }^{10}$ Interdisciplinary Graduate School of Medicine and Engineering, University of Yamanashi, Kofu, Yamanashi, Japan

${ }^{11}$ Academic Assembly School of Science and Technology Institute of Engineering, Shinshu University, Nagano, Nagano, Japan

12 The Graduate School of Science and Engineering, Saitama University, Saitama, Saitama, Japan

${ }^{13}$ Astrophysical Big Bang Laboratory, RIKEN, Wako, Saitama, Japan

${ }^{14}$ Department of Physics, SungKyunKwan University, Jang-an-gu, Suwon, Korea

${ }^{15}$ Department of Physics, Tokyo City University, Setagaya-ku, Tokyo, Japan

${ }^{16}$ Institute for Nuclear Research of the Russian Academy of Sciences, Moscow, Russia

${ }^{17}$ Faculty of Systems Engineering and Science, Shibaura Institute of Technology, Minato-ku, Tokyo, Japan

${ }^{18}$ Department of Engineering Science, Faculty of Engineering, Osaka Electro-Communication University, Neyagawa-shi, Osaka, Japan

${ }^{19}$ Department of Physics, Chiba University, Chiba, Chiba, Japan

${ }^{20}$ Service de Physique Théorique, Université Libre de Bruxelles, Brussels, Belgium

${ }^{21}$ Department of Physics, Yonsei University, Seodaemun-gu, Seoul, Korea

${ }^{22}$ Center for Astrophysics and Cosmology, University of Nova Gorica, Nova Gorica, Slovenia

${ }^{23}$ Faculty of Science, Kochi University, Kochi, Kochi, Japan

${ }^{24}$ Nambu Yoichiro Institute of Theoretical and Experimental Physics, Osaka City University, Osaka, Osaka, Japan

${ }^{25}$ Department of Physical Sciences, Ritsumeikan University, Kusatsu, Shiga, Japan

${ }^{26}$ Quantum ICT Advanced Development Center, National Institute for Information and Communications Technology, Koganei, Tokyo, Japan

${ }^{27}$ Sternberg Astronomical Institute, Moscow M.V. Lomonosov State University, Moscow, Russia

${ }^{28}$ Department of Physics, School of Natural Sciences, Ulsan National Institute of Science and Technology, UNIST-gil, Ulsan, Korea

${ }^{29}$ Earthquake Research Institute, University of Tokyo, Bunkyo-ku, Tokyo, Japan

${ }^{30}$ Graduate School of Information Sciences, Hiroshima City University, Hiroshima, Hiroshima, Japan

${ }^{31}$ Institute of Particle and Nuclear Studies, KEK, Tsukuba, Ibaraki, Japan

${ }^{32}$ Graduate School of Science and Engineering, Tokyo Institute of Technology, Meguro, Tokyo, Japan

${ }^{33}$ Department of Research Planning and Promotion, Quantum Medical Science Directorate, National Institutes for Quantum and Radiological Science and Technology, Chiba, Chiba, Japan

${ }^{34}$ CEICO, Institute of Physics, Czech Academy of Sciences, Prague, Czech Republic

${ }^{35}$ Department of Physics and Institute for the Early Universe, Ewha Womans University, Seodaaemun-gu, Seoul, Korea

\footnotetext{
* Deceased
} 